\title{
VARIAÇÃO SAZONAL DA CONCENTRAÇÃO DE MACRONUTRIENTES EM FOLHAS DE DIFERENTES GENÓTIPOS DE ACEROLEIRA ${ }^{1}$
}

\author{
ROSIANE DE LOURDES SILVA DE LIMA², DALMO LOPES DE SIQUEIRA ${ }^{3}$, JAIRO OSVALDO CAZETTA $^{4}$, \\ GILVAN BARBOSA FERREIRA ${ }^{5}$, OLMAR BALLER WEBER ${ }^{6}$.
}

RESUMO- A aceroleira produz durante o ano inteiro, e seus frutos são ricos em vitaminas e sais minerais. Para a obtenção de produções elevadas e frutos de boa qualidade, é necessário conhecer as necessidades nutricionais e as épocas mais apropriadas para a adubação das plantas. Este trabalho, conduzido em área do Campo Experimental da Embrapa Agroindústria Tropical, em Pacajus, Ceará, de dezembro de 1999 a outubro de 2000, teve por objetivo estudar a variação sazonal dos teores de nutrientes (N, P, K, Ca, Mg e S) e determinar a época mais indicada para a análise foliar de seis genótipos (P 52, P 66, P 78, P 91, P 93 e P 97) de aceroleira (Malpighia emarginata D.C.). Adotou-se o delineamento de blocos ao acaso, em esquema de parcelas subdivididas no tempo, com 4 repetições e uma planta por unidade experimental. Existem poucas diferenças no teor de macronutrientes das folhas de distintos genótipos de aceroleiras. O mês de dezembro é a época mais apropriada para diagnosticar os teores de macronutrientes em genótipos de aceroleira no Estado do Ceará, pois é o período menos influenciado pela falta ou excesso de chuva ou pela demanda dos órgãos em formação na planta.

Termos para indexação: Malpighia emarginata, nutrição mineral, época de amostragem.

\section{SEASONAL CHANGES OF NUTRIENT CONCENTRATION IN LEAVES OF DIFFERENT BARBADOS CHERRY GENOTYPES}

\begin{abstract}
Plants of Barbados cherry (Malpighia emarginata) produces all over the year, and its fruits are rich in vitamins and minerals. In order to obtain good production and high fruit quality it is necessary to know the nutritional need and the suitable season for plant fertilization. Thus, it was carried out an experiment at the EMBRAPA-National Center for Research of Tropical Agro-industry, from December 1999 to October 2000, aiming to study the seasonal changes nutrient (N, P, K, Ca, Mg and S) levels, and the suitable period for leaves mineral diagnosis, in six genotypes P 52, P 66, P 78, P 91, P 93 and P 97) and six distinct sampling times. All the 36 treatments (six genotypes evaluated in six harvest times) were conducted in a Randomized Completed Block (RCB) design with, and analyzed statistically as a split plot, with four replications composed by one plant each. It was observed low variations on macronutrient levels in the leaves among genotypes. December is the most suitable period for leaves sampling aiming to plant nutritional diagnosis of Malpighia emarginata, at Ceará Estate, Brazil.
\end{abstract}

Index terms: Malpighia emarginata, mineral nutrition, leaves sampling,time.

\section{INTRODUÇÃO}

A aceroleira (Malpighia emarginata D.C) é uma espécie tipicamente tropical, originária do Mar das Antilhas, sendo também encontrada na América Central, bem como no Norte e Noroeste da América do Sul (Visentainer et al., 1997), que vem sendo cultivada comercialmente no Brasil, principalmente nas regiões Sudeste e Nordeste (Cordeiro, 2000). Dentre os fatores responsáveis pela expansão do cultivo desta espécie, destacamse a riqueza natural em vitamina $\mathrm{C}$ diagnosticada em seus frutos (Alves, 1993; Cordeiro, 2000), a produção durante praticamente o ano todo (Nogueira, 1997; Amaral, 1998; Cordeiro, 2000) e o grande potencial de exportação da polpa congelada (Alves, 1993;
Nogueira,1997). Apesar de sua grande importância, destacada pelo constante crescimento nas áreas de cultivo (Paiva, 1996), há escassez de informações que auxiliem no planejamento das recomendações de adubação para os diversos genótipos desta espécie.

As pesquisas em torno da aceroleira têm-se concentrado principalmente nas áreas de propagação (Braga et al., 1997; Alloufa e Campos, 1999) e melhoramento genético (Lopes, 1999; Paiva et al., 1999; Cordeiro, 2000), especialmente visando à definição de variedades comerciais mais produtivas e com características organolépticas de frutos comercialmente aceitáveis pelo mercado consumidor. Porém, para a obtenção de produções elevadas e frutos de boa qualidade, é necessário o conhecimento das exigências nutricionais exibidas pelos genótipos (Lopes, 1999;

1 (Trabalho 188-06) Recebido em:17-11-2006. Aceito para publicação em: 26-10-2007. Parte da tese apresentada, pelo primeiro autor, à Universidade
Estadual Paulista (Unesp/Jboticabal) para obtenção do título de "Doctor Scientiae" em Produção Vegetal
${ }^{2}$ Enga Agron. Ms. Doutoranda em Agronomia (Produção Vegetal) - Unesp - Universidade Estadual Paulista - Jaboticabal-SP. limarosiane@yahoo.com.br.
${ }^{3}$ Prof. Adjunto, D.S., - Universidade Federal de Viçosa, Departamento de Fitotecnia, 36571-000, Viçosa-MG. siqueira@ufv.br.
${ }^{4}$ Prof. Adjunto, Unesp - Universidade Estadual Paulista, Departamento de Tecnologia, 14884-900, Jaboticabal-SP. cazetta@fcav.unesp.br .
${ }^{5}$ Eng $^{\circ}$ Agron. D.Sc. Pesquisador da Embrapa Roraima.Br 174, km 08, Distrito Industrial, C.P. 133, Boa Vista -RR, 69.301-970. gilvanbf@cpafrr.embrapa.br
${ }^{6}$ Eng $^{\circ}$ Agron. D.Sc. Pesquisador da Embrapa Agroindústria Tropical. Av. Dra. Sara Mesquita, 2270. Pici, Fortaleza-CE, 60511-110. weber@cnpat.embrapa.br

Rev. Bras. Frutic., Jaboticabal - SP, v. 29, n. 3, p. 652-656, Dezembro 2007 
Cordeiro, 2000) de aceroleira desenvolvidas pela pesquisa. Contudo, esta linha de pesquisa tem recebido pouca atenção por parte dos pesquisadores (Amaral, 1998; Silva, 1998), favorecendo o aparecimento de desordens nutricionais, redução na vida póscolheita dos frutos, baixa produtividade e frutos com características agronômicas desfavoráveis.

Para minimizar o quadro supracitado, o estudo das variações sazonais dos teores dos minerais e a definição de épocas apropriadas para o diagnóstico do estado nutricional dos diferentes genótipos cultivados poderão servir de critério para comparar os níveis de nutrientes nos tecidos da planta com a produtividade obtida e ajustar melhor a recomendação de adubação a ser utilizada pelo produtor. A comparação permitirá uma possível adequação de quantidades de fertilizantes que devem ser fornecidas e as épocas de maior consumo de nutrientes pelas plantas.

Neste contexto, esta pesquisa teve como objetivos avaliar a variação sazonal dos teores de $\mathrm{N}, \mathrm{P}, \mathrm{K}, \mathrm{Ca}, \mathrm{Mg}$ e $\mathrm{S}$ no tecido foliar e determinar a época mais indicada para a análise foliar, visando a obter informações que possam auxiliar na recomendação de fertilizantes nos estádios vegetativo e reprodutivo de genótipos de aceroleira.

\section{MATERIAL E MÉTODOS}

O ensaio foi conduzido em uma coleção de genótipos de aceroleira da Embrapa Agroindústria Tropical, em Pacajus-CE, no período de outubro de 1999 a outubro de 2000.

Adotou-se o delineamentoem blocos ao acaso, com quatro repetições, sendo que os tratamentos foram arranjados em esquema de parcela subdividida, tendo nas parcelas os efeitos de seis genótipos (P 52, P 66, P 78, P 91, P 93 e P 97) e nas subparcelas as 6 épocas de avaliação (meses do ano agrícola 1999-2000: dezembro/1999, fevereiro, abril, junho, agosto e outubro/2000). Os genótipos foram selecionados em um pomar de plantasmatrizes propagadas por sementes, conforme as características das plantas, que tinham os atributos desejados de conformação de copa, e o teor de vitamina $\mathrm{C}$ constatado nos frutos.

O latossolo Vermelho-Amarelo usado era arenoso e possuía baixa fertilidade, apresentando acidez elevada $(\mathrm{pH} 4,8)$ e alumínio trocável em baixo teor $\left(0,15 \mathrm{mmol}_{\mathrm{c}} \mathrm{dm}^{-3}\right)$; a saturação de bases apresentava-se relativamente elevada $(89 \%)$, baixo teor de $\mathrm{P}\left(6,4 \mathrm{mg} \mathrm{dm}^{-3}\right)$, médio teor de $\mathrm{K}\left(1,5 \mathrm{mmol}_{\mathrm{c}} \mathrm{dm}^{-3}\right)$, baixo teor de matéria orgânica $\left(11,48 \mathrm{~g} \mathrm{~kg}^{-1}\right)$, com o teor de Ca no nível de 5,3 $\mathrm{mmol}_{\mathrm{c}} \mathrm{dm}^{-3} \mathrm{e} \mathrm{Mg}$ de 3,0 $\mathrm{mmol}_{\mathrm{c}} \mathrm{dm}^{-3}$. Entre os meses de dezembro de 1999 e outubro de 2000 , foram registrados $1.122,2 \mathrm{~mm}$ de chuva, sendo que, nos meses de janeiro a maio de 1999, choveu $845 \mathrm{~mm}$. $\mathrm{O}$ menor volume de chuva foi registrado nos meses de setembro e outubro de 2000 (36,1 e 2,0 mm). A quantidade de chuva foi considerada satisfatória para o pleno desenvolvimento da cultura.

As plantas foram cultivadas em regime de sequeiro, no espaçamento $3 \mathrm{~m} \mathrm{x} 4 \mathrm{~m}$, totalizando 833 plantas/ha. A fertilização das plantas foi realizada a cada dois meses, sendo que, nos meses de outubro e dezembro de 1999, cada planta recebeu $90 \mathrm{~g}$ de uréia, $72 \mathrm{~g}$ de cloreto de potássio e $20 \mathrm{~g}$ de sulfato de magnésio e, nos meses de janeiro, março, maio, julho, agosto e outubro de 2000, a adubação foi com $30 \mathrm{~g}$ de uréia, $50 \mathrm{~g}$ de monoamoniafosfato (MAP), $20 \mathrm{~g}$ de sulfato de magnésio e $40 \mathrm{~g}$ de cloreto de potássio, aplicando-se em sulcos rasos, abertos em meia-lua na projeção da copa.

Durante a condução do experimento, amostras foliares foram coletadas a cada 60 dias (meses de dezembro de 1999 e fevereiro, abril, junho, agosto e outubro de 2000), no terço mediano dos ramos localizados na porção superior da copa das plantas.

As folhas amostradas foram identificadas, acondicionadas em sacos de papel, lavadas com água deionizada (Jones Júnior et al., 1991) e secas em estufa com circulação forçada de ar, a $70^{\circ} \mathrm{C}$, até peso constante. Posteriormente, as amostras foram moídas em moinho tipo Willey, passadas em peneira de malha de 20 mesh, e acondicionadas em recipientes de polietileno com tampa hermética. Foram submetidas à digestão nítrico-perclórica para determinar as concentrações de $\mathrm{P}, \mathrm{K}, \mathrm{Ca}, \mathrm{Mg}$ e S. Para a determinação do teor de $\mathrm{N}$, as amostras foram submetidas à digestão sulfúrica. $\mathrm{ON}$ foi determinado pelo método colorimétrico de Nessler, o P pelo método da redução do fosfomolibdato pela vitamina C, modificado por Braga e Delfelipo (1974), e o K por fotometria de chama. $\mathrm{O} \mathrm{Ca}$ e o $\mathrm{Mg}$ foram determinados por espectrofotometria de absorção atômica, e o S por turbidimetria do sulfato (Blanchar et al., 1963).

Os dados coletados foram submetidos à análise de variância, pelo teste $\mathrm{F}$, e as diferenças entre os fatores qualitativos foram mensuradas nas variáveis através da aplicação do teste de múltipla comparação de médias (Tukey), ao nível de 5\% de probabilidade.

\section{RESULTADOS E DISCUSSÃO}

Os teores foliares de macronutrientes nas diferentes épocas de avaliação diferiram significativamente entre os genótipos estudados, observando-se efeito significativo para épocas de amostragem para os nutrientes $\mathrm{N}$ total e $\mathrm{Mg}$, e interação significativa entre os fatores genótipos e épocas de amostragem para os nutrientes K, Ca e S, respectivamente (Tabela 1 .

Foram detectadas diferenças significativas para os teores foliares de $\mathrm{NH}_{4}^{+}$e $\mathrm{N}$-total nas épocas estudadas, não se observando diferenças significativas entre os genótipos. Observou-se aumento nos teores deste nutriente de dezembro/ 99 para fevereiro/00, (Tabela 2), reduzindo sensivelmente nas amostragens efetuadas de abril a outubro/ 00 . O maior teor de Ntotal foi observado na amostragem de fevereiro, correspondendo a 30,5 $\mathrm{g} \mathrm{kg}^{-1}$, e mínimo de $21,8 \mathrm{~g} \mathrm{~kg}^{-1}$, na amostragem de ago/00.

$\mathrm{O}$ acréscimo nos teores foliares de $\mathrm{N}$ de dezembro/99 para fevereiro/00, possivelmente, está relacionado com a adubação nitrogenada realizada neste período. Embora a planta se encontrasse em pleno ciclo reprodutivo, a demanda por $\mathrm{N}$ das fontes (folhas) para os drenos (frutos) foi minimizada pela adição deste elemento no solo através da adubação nitrogenada. A menor concentração de $\mathrm{N}$ observada a partir de fevereiro pode ser explicada pelo maior florescimento e frutificação promovidos pela intensificação das chuvas, ocorridas neste período, o que deve ter aumentado a demanda e a translocação do nutriente para os frutos. 
Na primeira amostragem, constatou-se que os teores de $\mathrm{N}$-total se encontravam acima dos níveis críticos de 20 a $24 \mathrm{~g} \mathrm{~kg}^{-}$ ${ }^{1}$ comparados com os verificados por Oliveira (2004) e por Andrade (2004). Para plantas de aceroleira, são considerados adequados teores de 20 a $26 \mathrm{~g} \mathrm{~kg}^{-1}$ (Silva, 1998).

Não se constataram diferenças significativas para genótipos dentro de épocas de avaliação. Como o nitrogênio é um nutriente de alta mobilidade no floema (Marschner, 1995), essas variações foram atribuídas à translocação deste nutriente das folhas maduras para as jovens, para as flores e, preferencialmente, para os frutos, uma vez que a aceroleira apresenta flores e frutos em vários estágios de desenvolvimento, simultaneamente (Cordeiro, 2000).

Quanto ao $\mathrm{Mg}$, observou-se apenas efeito de época para este elemento, não se observando diferenças significativas para genótipos. Os teores de $\mathrm{Mg}$ (Tabela 2) apresentaram-se abaixo da faixa observada por Jones Júnior et al. (1991) e Amaral (1998), mas dentro dos padrões estabelecidos por Oliveira (2004) e Andrade (2004) (1,5 a 2,5 $\mathrm{g} \mathrm{kg}^{-1} \mathrm{e}$ de 3 a $\left.4 \mathrm{~g} \mathrm{~kg}^{-1}\right)$, respectivamente. $\mathrm{O} \mathrm{Mg}$ apresentou oscilação acentuada no decorrer das avaliações, com valor mínimo de $3,7 \mathrm{~g} \mathrm{~kg}^{-1}$ na amostragem de junho/00, e máximo de 5,1 $\mathrm{g} \mathrm{kg}^{-1}$ na amostragem de dezembro/99. Marinho et al. (2002) relatam que o $\mathrm{Mg}$ é um nutriente absorvido com maior intensidade nos meses quentes de verão, diminuindo a absorção no outono e no inverno. Os dados desta pesquisa, no entanto, mostram maiores teores de $\mathrm{Mg}$ no período seco, e menores no período chuvoso, onde verificaram-se alteas taxas de crescimentos. Para a aceroleira, tudo indica que existe o efeito de competição pelas doses de $\mathrm{K}$ e de diluição e concentração pela resposta da planta ao período de umidade. Os teores de $\mathrm{Mg}$ no solo, menores que 09 mmol $\mathrm{dm}^{-3}$, caracterizam solo com deficiência neste elemento (Piza e Guaggio, 1996), o que pode explicar os baixos teores medidos na folha.

Os teores de $\mathrm{P}$ não revelaram diferenças significativas entre épocas nem entre genótipos (Tabela 1). Assim sendo, no que se refere ao $\mathrm{P}$, qualquer época seria adequada para se avaliar o estado nutricional das plantas. Os valores encontrados neste trabalho apresentaram-se acima dos níveis de suficiência verificados por Oliveira (2004) e Andrade (2004), em plantas de aceroleira (0,8 a $\left.1,2 \mathrm{~g} \mathrm{~kg}^{-1}\right)$, bem como por Amaral (1998) (1,2 a 2,2 $\left.\mathrm{g} \mathrm{kg}^{-1}\right)$. Os teores foliares de P variaram entre $1,27 \mathrm{~g} \mathrm{~kg}^{-1}$ e $2,03 \mathrm{~g} \mathrm{~kg}^{-1}$, valores observados nas amostragens de dezembro de 1999 e abril de 2000.

Quanto ao potássio (Tabela 3), detectou-se diferenças significativas na habilidade em concentrar $\mathrm{K}$ nas folhas entre genótipos, nas várias épocas de amostragem, verificando-se, em geral, maior concentração deste nutriente nas amostragens de dez/99 e fev/00. Observaram-se teores foliares máximos de K da ordem de 29,9 $\mathrm{g} \mathrm{kg}^{-1}$ em tecidos foliares do genótipo P 66 na amostragem de dezembro/99 e mínimo de 9,3 $\mathrm{g} \mathrm{kg}^{-1} \mathrm{em}$ tecidos foliares do genótipo P 78 na amostragem de outubro/00. Amaral (1998) constatou teores oscilando de 15,8 a $25,3 \mathrm{~g} \mathrm{~kg}^{-1} \mathrm{em}$ plantas adultas de aceroleira, que está dentro da variação observada nesta pesquisa. Por outro lado, os teores mínimos de K encontravamse abaixo do nível inferior proposto por Oliveira (2004) e Andrade (2004) em plantas de aceroleira, que eram de 15 a $20 \mathrm{~g} \mathrm{~kg}^{-1} \mathrm{e}$ de 22 a $30 \mathrm{~g} \mathrm{~kg}^{-1}$, respectivamente. As concentrações foliares de $\mathrm{K}$ detectadas encontraram-se dentro do nível de suficiência (15 - 30 $\mathrm{g} \mathrm{kg}^{-1}$ ), de acordo com Silva (1998); sendo assim, não foi observada deficiência de $\mathrm{K}$ nos genótipos estudados.

De acordo com Amaral (1998), o menor teor foliar deste nutriente ocorreu no final de novembro e início de dezembro. Contudo, os dados exibem tendências contrárias. É possível que as condições climáticas observadas no município de Visconde do Rio Branco, em Minas Gerais, tenham propiciado as diferenças encontradas pelo autor supracitado, e ainda indicando menores variações que as verificadas entre os genótipos e nas amostragens de abril, junho, agosto e outubro/2000, épocas de intenso florescimento e frutificação. Aparentemente, a aceroleira cultivada em regime de sequeiro teve tempo para carregar seus tecidos de $\mathrm{K}$ no início do período chuvoso (fevereiro/00) e, em seguida, devido à intensa translocação de $\mathrm{K}$ para os frutos, que é um dreno forte desse nutriente, a concentração diminui até o mínimo necessário para suas atividades metabólicas sem estresse nutricional.

Observando-se os valores da Tabela 4, verifica-se que houve interações significativas para os teores foliares de $\mathrm{Ca}$, entre genótipos e épocas de amostragem. Os teores de Ca mantiveramse abaixo das concentrações indicadas como adequadas por Jones Júnior et al. (1991) e Amaral (1998) (25,9 $\mathrm{g} \mathrm{kg}^{-1} ; 15-25 \mathrm{~g} \mathrm{~kg}^{-1}$, respectivamente), em todas as épocas de amostragem. Esse baixo teor pode ser a conseqüência do uso de doses elevadas de $\mathrm{K}$ em solo arenoso com baixos teores de Ca. Outro aspecto que deve ser levado em conta, é a de que o $\mathrm{K}$ pode estar interferindo negativamente na absorção do Ca (Marscnher, 1995).

O teor máximo $\left(9,8 \mathrm{~g} \mathrm{~kg}^{-1}\right)$ foi diagnosticado nos tecidos foliares do genótipo P 91, na amostragem de outubro/00, e a menor concentração foi constatada em tecidos foliares do genótipo P 52 $\left(3,6 \mathrm{~g} \mathrm{~kg}^{-1}\right)$, na amostragem de junho/00. Os menores teores médios foram diagnosticados nas amostragens de fevereiro/ $00 \mathrm{e}$ abril/ $/ 00$. As maiores concentrações de $\mathrm{Ca}$ entre junho e dezembro podem estar relacionadas à idade das folhas das plantas, pois o Ca é um elemento de baixa mobilidade no floema (Marschner, 1995) e, portanto, sua concentração tende a aumentar com a idade das folhas, embora o período avaliado seja pequeno.

Os teores foliares de $\mathrm{S}$ foram bastante estáveis na amostragem de dezembro/99 e outubro/00 (Tabela 5), mantendose abaixo da faixa de 4 a $6 \mathrm{~g} \mathrm{~kg}^{-1}$, considerada satisfatória por Oliveira (2004). Os menores teores de $\mathrm{S}\left(0,9 \mathrm{~g} \mathrm{~kg}^{-1}\right)$ foram verificados nas amostragens de junho, e os maiores, na amostragem de dezembro/99, seguidos pelos de outubro/00, valores médios de $2,3 \mathrm{~g} \mathrm{~kg}^{-1}$. Em ambas as épocas, ocorreram variações nos teores de $\mathrm{S}$ entre os genótipos, destacando-se os genótipos $\mathrm{P} 66$ na amostragem de dezembro e a P 97 na amostragem de outubro, por apresentar maiores teores médios de $\mathrm{S}$ na massa seca foliar das árvores.

Em geral, houve mais variabilidade entre as épocas de amostragem do que entre os genótipos. Isto indica a possibilidade da seleção de uma única data de amostragem para se obter boa estimativa do estado nutricional das plantas, sendo o mês de dezembro a época indicada para análise foliar da aceroleira. 
TABELA 1- Resumo da análise de variância dos teores foliares de $\mathrm{NH}_{4}$, N-total, $\mathrm{P}, \mathrm{K}, \mathrm{Ca}, \mathrm{Mg}$ e S de genótipos de aceroleira, em seis épocas de avaliação. PacajusCE, 1999-2000.

\begin{tabular}{lllllllll}
\hline \multirow{2}{*}{ F. V. } & \multirow{2}{*}{ G.L. } & \multicolumn{7}{c}{ Quadrados médios } \\
\cline { 3 - 9 } & & $\mathrm{NH}_{4}$ & $\mathrm{~N}$ Notal & $\mathrm{P}$ & $\mathrm{K}$ & $\mathrm{Ca}$ & $\mathrm{Mg}$ & $\mathrm{S}$ \\
\hline Bloco & 3 & 0,46 & 1,30 & 0,89 & 55,02 & 0,82 & 0,43 & 0,18 \\
Progênie & 5 & 14,81 & 16,54 & 1,40 & 170,18 & $5,30^{*}$ & 0,98 & 0,33 \\
Erro (a) & 15 & 19,67 & 24,74 & 0,78 & 153,06 & 1,74 & 1,19 & 0,34 \\
Época & 5 & $181,08^{*}$ & $242,54^{*}$ & 1,81 & $257,44^{*}$ & $47,57^{*}$ & $6,44^{*}$ & $2,74^{*}$ \\
Px E & 25 & 4,96 & 5,63 & 0,79 & $24,86^{*}$ & $5,92^{*}$ & 0,36 & $0,12^{*}$ \\
Erro (b) & 90 & 6,88 & 8,02 & 0,83 & 12,90 & 1,25 & 0,21 & 0,06 \\
CV (a) & - & 18,6 & 20,2 & 49,6 & 68,0 & 22,1 & 25,5 & 41,4 \\
CV (b) & - & 11,0 & 11,5 & 51,2 & 19,7 & 18,7 & 10,7 & 17,4 \\
\hline
\end{tabular}

* Significativo a 1 e $5 \%$, pelo Teste $\mathrm{F}$.

TABELA 2 - Teores médios de $\mathrm{NH}_{4}$, N-total e $\mathrm{Mg}\left(\mathrm{g} \mathrm{kg}^{-1}\right)$ na massa seca foliar de genótipos de aceroleira, em seis épocas de amostragem. Pacajus-CE, 1999-2000.

\begin{tabular}{|c|c|c|c|}
\hline ÉPOCA & $\mathrm{NH}_{4}$ & N Total & $\mathrm{Mg}$ \\
\hline Dezembro & $25,3 \quad b$ & $25,6 \quad b$ & $5,1 \mathrm{a}$ \\
\hline Fevereiro & $28,8 \mathrm{a}$ & $30,5 \mathrm{a}$ & $4,1 \mathrm{c}$ \\
\hline Abril & 23,3 bc & 24,0 bc & $4,0 \mathrm{~cd}$ \\
\hline Junho & 22,5 & 23,2 & $3,7 \quad d$ \\
\hline Agosto & 21,4 & 21,8 & $4,1 \mathrm{~cd}$ \\
\hline Outubro & 22,1 & 22,4 & $4,6 \mathrm{~b}$ \\
\hline
\end{tabular}

Médias de época seguidas das mesmas letras não diferem entre si, pelo teste de Tukey $(\mathrm{p}<0,05)$.

TABELA 3 - Teores médios de $\mathrm{K}\left(\mathrm{g} \mathrm{kg}^{-1}\right)$ na massa seca foliar de genótipos de aceroleira, em seis épocas de amostragem. Pacajus-CE, 1999-2000

\begin{tabular}{lllllll}
\hline \multirow{2}{*}{ Progênie } & \multicolumn{7}{c}{ Épocas } \\
\cline { 2 - 7 } 52 & Dezembro & Fevereiro & Abril & Junho & Agosto & Outubro \\
\cline { 2 - 7 } 66 & A 17,6 a & A 22,4 a & A 17,9 a & A 16,0 a & A 18,5 a & A 16,4 a \\
78 & A 29,9 a & A 28,6 a & B 17,3 a & B 16,7 a & B 16,6 a & B 18,1 a \\
91 & B 11,4 b & A 23,7 a & B 12,9 a & B 12,7 a & B 09,7 a & B 09,3 a \\
93 & A 18,2 a & A 22,4 a & A 18,2 a & A 17,7 a & A 17,7 a & A 15,8 a \\
97 & AB 17,9 a & A 25,7 a & B 18,2 a & B 18,1 a & AB 18,8 a & AB 19,1 a \\
& A 24,3 a & B 16,7 a & B 15,8 a & AB 17,7 a & AB 18,0 a
\end{tabular}

Médias de época seguidas das mesmas letras, maiúscula na linha e minúscula na coluna, não diferem entre si, pelo teste de Tukey $(\mathrm{p}<0,05)$.
TABELA 4 - Teores médios de $\mathrm{Ca}\left(\mathrm{g} \mathrm{kg}^{-1}\right)$ na massa seca foliar de genótipos de aceroleira, em seis épocas de amostragem. Pacajus-CE, 1999-2000.

\begin{tabular}{lllllll}
\hline \multirow{2}{*}{ Progênie } & \multicolumn{5}{c}{ Épocas } \\
\cline { 2 - 7 } & Dezembro & Fevereiro & Abril & Junho & Agosto & Outubro \\
\hline 52 & AB 5,6 ab & B 3,8 a & B 4,0 a & B 3,6 b & A 6,4 a & A 7,8 a \\
66 & A 7,8 a & B 4,9 a & B 5,2 a & B 5,2 a & A 8,6 a & B 4,4 b \\
78 & AB 6,7 ab & C 4,3 a & BC 4,7 a & ABC 6,5 a & A 8,8 a & BC 4,7 b \\
91 & BC 5,6 ab & C 4,2 a & C 4,8 a & B 7,4 a & AB 7,7 a & A 9,8 a \\
93 & BC 5,4 b & C 4,3 a & BC 4,6 a & AB 6,7 a & A 8,3 a & A 8,7 a \\
97 & A 5,4 b & B 3,8 a & B 4,4 a & A 6,8 a & A 7,2 a & A 7,7 a \\
\hline
\end{tabular}

Médias de época seguidas das mesmas letras maiúscula na linha minúscula na coluna não diferem entre si, pelo teste de Tukey $(\mathrm{p}<0,05)$.

TABELA 5 - Teores médios de $\mathrm{S}\left(\mathrm{g} \mathrm{kg}^{-1}\right)$ na massa seca foliar de genótipos de aceroleira, em seis épocas de amostragem. Pacajus-CE, 1999-2000.

\begin{tabular}{lllllll}
\hline \multirow{2}{*}{ Progênie } & & \multicolumn{5}{c}{ Épocas } \\
\cline { 3 - 6 } & Dezembro & Fevereiro & Abril & Junho & Agosto & Outubro \\
\hline 52 & A2,0 a & B 1,4 a & B 1,3 a & B 1,2 a & B 1,3 a & AB 1,5 b \\
66 & A 2,3 a & B 1,2 a & B 1,0 a & B 1,1 a & B 1,3 a & B 1,4 b \\
78 & AB 1,8 a & C 1,3 a & C 1,1 a & C 1,1 a & BC 1,3 a & A 1,9 ab \\
91 & A 2,0 a & BC 1,4 a & C 1,2 a & C 1,0 a & BC 1,4 a & AB 1,8 ab \\
93 & A 1,6 a & ABC 1,2 a & BC 1,1 a & C 0 1,9 a & ABC 1,2 a & ABC 1,5 b \\
97 & AB 2,0 a & C 1,2 a & C 1,2 a & C 1,3 a & BC 1,6 a & A 2,3 a
\end{tabular}

Médias de época ou genótipo seguidas das mesmas letras, maiúscula na linha e minúscula na coluna, não diferem entre si, pelo teste de Tukey $(\mathrm{p}<0,05)$.

\section{CONCLUSÕES}

1-Existem poucas diferenças no teor de macronutrientes das folhas de distintos genótipos de aceroleiras.

2-O mês de dezembro é a época mais apropriada para diagnosticar os teores de macronutrientes em genótipos de aceroleira no Estado do Ceará, pois é o período em que são menos influenciados pela falta ou pelo excesso de chuva ou ainda, pela demanda dos órgãos em formação na planta. 


\section{REFERÊNCIAS}

ALLOUFA, M.A.I.; CAMPOS, M.A. Calogênese de acerola (Malpighia glaba. L.) a partir de explantes foliares cultivados in vitro. Revista Brasileira de Fruticultura, Jaboticabal, v.21, n.3, p.284-287, 1999.

ALVES, R.E. Acerola (Malpighia emarginata D.C.) fisiologia da maturação e armazenamento refrigerado sob atmosfera ambiente e modificada. 1993. 99 f. Dissertação (Mestrado em Fisiologia Vegetal) - Escola Superior de Agricultura de Lavras, Lavras, 1993.

AMARAL, J.F.T. do. Parte da planta e época para diagnose do estado nutricional e crescimento de ramos em aceroleira (Malpighia emarginata D.C.). 1998. Dissertação (Mestrado em Fitotecnia)- Universidade Federal de Viçosa, Viçosa, 1998.

ANDRADE, L.R.M. Corretivos e fertilizantes para culturas perenes e semiperenes. In: SOUSA, D.M.G.; LOBATO, R. (Eds.). Cerrado: correção do solo e adubação. 2.ed. Brasília: Embrapa Informação Tecnológica, 2004. p.317-366.

BLANCHAR, R.W.; REHM, G.; CALDWELL,A.C. Sulfur in plant material by digestion with nitric and perchloric acid. Proceedings of the Soil Science Society of America, Madison, v. 29, p.71-72, 1963.

BRAGA, M. F.; CALDAS, L.S.; HABE, M.H. Estabelecimento de acerola (Malpighia glaba. L.) in vitro: efeito do clone e explante. Revista Brasileira de Fruticultura, Cruz das Almas, v.19, n.3, p.335-346, 1997.

BRAGA, J.M.; DEFELIPO, B.V. Determinação espectrofotométrica de P em extratos de solo e material vegetal. Revista Ceres, Viçosa, v.21, n.113, p.73-85, 1974.

CORDEIRO, E.R. Seleção de genótipos de polinização livres e estimativas de parâmetros genéticos em acerola (Malpighia emarginata D.C.). 2000. Dissertação (Mestrado em Fitotecnia)Universidade Federal do Ceará, Fortaleza, 2000.

JONES JÚNIOR, J.B.; WOLF, B.; MILLS, H.A. Plant analysis handbook: a practical sampling, preparation, analysis, and interpretation guide. Athens, Georgia: Micro-Macro Publishing, 1991.213p.

LOPES, R. Polimorfismo, sistema de acasalamento, polinizações, repetibilidade de características do fruto e avaliação de genótipos de aceroleira (Malpighia emarginata D.C.). 1999. Dissertação (Mestrado em genética e melhoramento)- Universidade Federal de Viçosa, Viçosa, 1999.
MARINHO, C.S.; MONNERAT, P.H; CARVALHO JÚNIOR, A.C. de.; MARTINS, S.L.D.;VIEIRA, A. Análise química do pecíolo e limbo foliar como indicadora do estado nutricional dos mamoeiros 'Solo'e 'Formosa'. Scientia Agrícola, Piracicaba, v.59, n.2, p.373$381,2002$.

MARSCNER, $H$. Mineral nutrition of higher plants. $2^{\text {nd }} e d$. London: Academic Press, 1995. 889p.

NOGUEIRA, R.J.M.C. Expressões fisiológicas da aceroleira (Malpighia emarginata D.C.) em condições adversas. 1997. 205f. Tese (Doutorado em ecologia e recursos naturais) - Universidade Federal de São Carlos, São Carlos, 1997.

OLIVEIRA, S.A de. Análise foliar. In: SOUZA, D.M.G., LOBATO, R (Eds). Cerrado: correção do solo e adubação. 2.ed. Brasília: Embrapa Informação Tecnológica, 2004. p.245-256.

PAIVA, J.R. Caso de la Acerola: de espécie silvestre a producto de demanda creciente em los mercados mundiales. In: PROGRAMA REGIONAL DE PROMOCCIÓN SUSTENIBLE Y UTILIZACION DE FRUTAS Y HORTALIZAS AMAZONICAS: ESTRATEGIAS E ACCIONES, 1996, Pucallpa. Trato de Cooperación Amazônica. Pucallpa: FAO, 1996. p.179-198.

PAIVA, J.R.; PAIVA, W.O.; CORDEIRO, E.R.; SABRY NETO, H. Parâmetros genéticos em genótipos de polinização livre de acerola. Pesquisa Agropecuária Brasileira, Brasília, v.34, n.4, 1999, p.629-634.

PIZA JR., C.; QUAGGIO, J.A. Acerola ou cereja-das-antilhas. In: RAIJ, B.VAN; CANHARELA, H.; QUAGGIO, J.A.; FURLANI, A.M.C. Recomendações de adubação e calagem para o estado de São Paulo. 2.ed. Campinas: Instituto Agronômico \& Fundação IAC, 1996. 258p. (Boletim Técnico 100).

SILVA, G.D. Marcha de absorção de macronutrientes e micronutrientes em aceroleira (Malpighia glaba. L.). 1998. Dissertação (Mestrado em Fitotecnia) - Universidade Federal de Viçosa, Viçosa, 1998.

VISENTAINER, J.V.; VIEIRA, O.A.; MATSUSHITA, M.; SOUZA, N.E. de. Caracterização físico-química da acerola Malpighia glaba L. produzida na região de Maringá, Estado do Paraná, Brasil. Archivos Latinoamericanos de Nutricion, Caracas, v.47, n.1, 1997. 